\title{
Date Time of Onset of Angina Pectoris
}

National Cancer Institute

\section{Source}

National Cancer Institute. Date Time of Onset of Angina Pectoris. NCI Thesaurus. Code C91346.

The date and time of the beginning of the symptom complex of angina pectoris. 